\title{
CRITICAL ANALYSIS OF RADAR DATA SIGNAL DE-NOISING BY IMPLEMENTATION OF HAAR WAVELET TRANSFORMATION
}

\author{
Ananth Abhishek Jillepalli ${ }^{1}$, Raghavendra Deeduvanu ${ }^{2}$, Sreenath DeviReddy ${ }^{3}$, Deepak Vangala ${ }^{4}$, Ravi \\ Mathey $^{5}$ \\ ${ }^{1} I V^{\text {th }}$ Year, Department of CSE, Vidya Jyothi Institute of Technology, Hyderabad \\ ${ }^{2} I V^{\text {th }}$ Year, Department of CSE, Vidya Jyothi Institute of Technology, Hyderabad \\ ${ }^{3} I V^{\text {th }}$ Year, Department of CSE, Vidya Jyothi Institute of Technology, Hyderabad \\ ${ }^{4} I V^{\text {th }}$ Year, Department of CSE, Vidya Jyothi Institute of Technology, Hyderabad \\ ${ }^{5}$ Head of Department, Department of Computer Science and Engineering, Vidya Jyothi Institute of Technology, \\ Hyderabad
}

\begin{abstract}
Data signal de-noising is a crucial part of every transaction which involves capturing waves and processing them into understandable format. Most essentially, this process of data signal de-noising is important in the field of military and defence. Because of the rapid development in military communication systems, a limitation arises in the systems. The limitation being quantity of signals profoundly increases along with noise and other disturbances' presence in the signal. In order to overcome this drawback, batch de-noising techniques are implemented on the systems to produce clean signals. In this paper, the critical analysis case of RADAR [RAdio Detection And Ranging] data de-noising by implementation of Haar Wavelet Transformation is considered. A signal set simulation containing four signals procured from RADAR data is taken into consideration. The signals are then subjected to de-noising in MATLAB and indigenously developed python tool using Haar Wavelet Transformations. The ensuing results pertaining to accuracy and efficiency of output signals are then compared.
\end{abstract}

Keywords: RADAR data, MATLAB tool, Python, De-noising techniques, Haar Wavelet Transformation, Signal Simulation.

\section{INTRODUCTION}

The main focus of this paper is to contrast de-noising methods available in two different tools and to determine the most efficient and accurate output producing tool. The potential conclusion for this analytical problem case will also include the extent of memory consumption by the two tools. To this point, the intention of this paper is to perform de-noising of four signals MATLAB and Python, in order to properly de-noise the signals, such that a military system can precisely and memory efficiently process the signals being received into understandable data. The substantial inspiration for this work is that the problem of efficient and accurate denoising targets considerable number of military tracking systems.

The process of de-noising a captured RADAR data set commences with the processing and selection of a single relevant signal from the entire captured set. The signal selection is of instrumental importance to the process of cleaning the signal and subsequently will be applied to entire set of signals. Our efforts enable us to optimise the process through which signals can be cleaned out efficiently without much data loss and precision, as intended. A potential conclusion and a result like this one will not only supplement military tracking and communication systems in understanding the signal data quickly and with agility, but it will also reduce system maintenance costs as our research also enables us to find out cost effective tool for de-noising. This paper will concentrate on the critical analysis of data signal de-noising from a signal set simulated from the data obtained from RADAR transceivers.

\section{BACKGROUND STUDY}

The large number of signals received in transceiver systems far outruns the modules deployed for denoising. We conducted some initial experiments on internal equipment and were able to deduce the most frequent category of signals received. Prior research on this matter revealed the four standard categories of signals being received frequently:

- $\quad$ First, the most frequent category of signals being received belongs to tracking type of data which track the whereabouts of any device and report back to the base.

- Second, the next frequent class of signals being received belongs to communication data type where in one base communicates with the other.

- Next, the signals being received in this category belong to medical data type of the military personnel which contain the medical records of the staff and soldiers.

- Finally, the least category of signals received is trash or pranks which are mostly useless. 


\subsection{Signal Overview}

Signals have many instances and forms depending upon the category of the data being transmitted. The overview of signal forms is as follows:

\section{Overview}

A very frequently found type of signal wave is the sine wave. These signals are used almost for every type of data transmission. Sine waves are also easier to de-noise since there many functions developed for sine owing to the huge potential of applications.

A not so frequently used type of signal wave is the Doppler wave. These signals are not used unless the data transmission must be very secure. Doppler waves are hard and slow to transmit and so, anyone who uses them should sacrifice speed for security.

A very rare type of signals wave used are the Chebyshev filter waves. The infrastructure needed to generate these waves is so complex that these waves were generated only one in entire human history till now. They are fast, secure and reliable but complex to generate.

\section{CONTEMPORARY USAGE OF DE-NOISING}

\section{IN VARIOUS SYTEMS}

The conventional process of eliminating unnecessary disturbances and any unwanted resources from an entity has been coined into a single term, known as de-noising. From then on, this phenomenon of de-noising an entity has been implemented on various fields and for various applications.

Technically, de-noising is taking any variable or entity as input and removing unwanted data, called "noise" with the use of some algorithms. The various implementations of de-noising functionality is as follows:

\subsection{Image Refining using De-Noising}

Unclear and obfuscated images are often not desirable for obvious reasons of lacking clarity. As such, to make these images clearer, there have been many attempts at developing image de-noising software. Some successful attempts resulted in software like Topaz Image Cleaner, Ninja Noise Remove and Photoshop Image processor.

\subsection{Data Refinement using De-noising}

Refinement of data, also known as data cleaning or rarely also known as data classification is the process of eliminating opaque data item sets from relevant material in a large collection of data items, like Data Warehouses and Data Bases.

Due to the numerous successful attempts of data denoising, made in the last decade, there are a couple of tools in market available, which can efficiently refine data without much data loss and with precision. Most popular ones are WEKA tool and CITRUS development environment which includes internally built data de-noising module.

\subsection{Signal Refining using De-noising}

Partly as a result of gigantic expansion of signal usage for data transmission in the last few decades, there has been an urgent need for some techniques which would be capable of eliminating unnecessary data and unwanted disturbances from the signals.

As such, there have been countless attempts made at developing algorithms and subsequently developing tools which can de-noise signals of any format and containing any data formats. As a result, there have arisen numerous tools for de-noising signals universally, though most of them are inefficient and even incompetent to some extent.

There are presently three tools which, we can classify under "working as intended" category. They are: MATLAB, OriginPro and Sony WavePad (for audio signals only). Of these three, only MATLAB and OriginPro are capable of de-noising signals of any format and type. And of those two, our experiments clearly showed that MATLAB produces accurate and efficient results.

\subsection{Proposed Tool}

The tool that we propose has been built from scratch using Python, VB Script and minor C++ functionality only. No external modules or third party software was used in the indigenous development of our tool. In our internal experiments, the python tool was able to de-noise signals of all formats and any data type. As such, we move forward to compare MATLAB and our tool on the basis of efficiency, accuracy, compatibility and memory costs.

\section{OBSERVATION AND ANALYSIS}

We have generated four signals containing RADAR data simulation. Three of which relate to tracking and communication type of data and one of them is a medical record type of data. The Sequence of our Observation and subsequent analysis is as follows:

- MATLAB Observation

- Python Tool Observations

- Comparative Analysis

\subsection{MATLAB Observations}

All the MATLAB Observations have been conducted through the use of a specialised module toolbox known as Wavelet Toolbox. The Signal Graphs shown below are only screenshots of MATLAB Wavelet toolbox results.

\subsubsection{Signal \#1}

Signal \#1 contains information which pertains to the data sent by trackers. Trackers are basic identifying devices which can be used to track any object. 


\section{Original Signal}

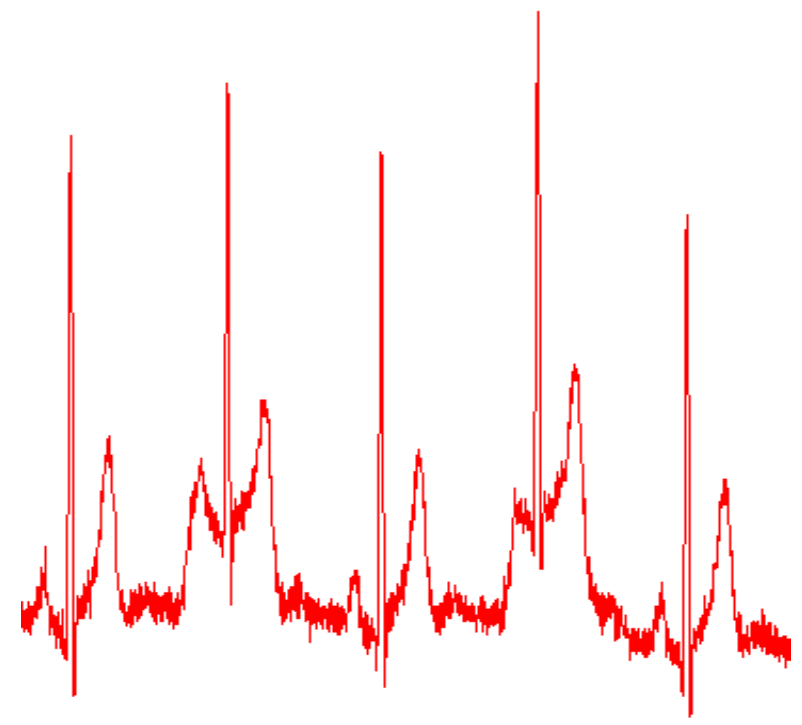

Fig 1: - Original Signal \#1 (MATLAB)

Frequency - 100 cycles / 1 sec Standard Deviation - 20 metres

\section{De-Noised Signal}

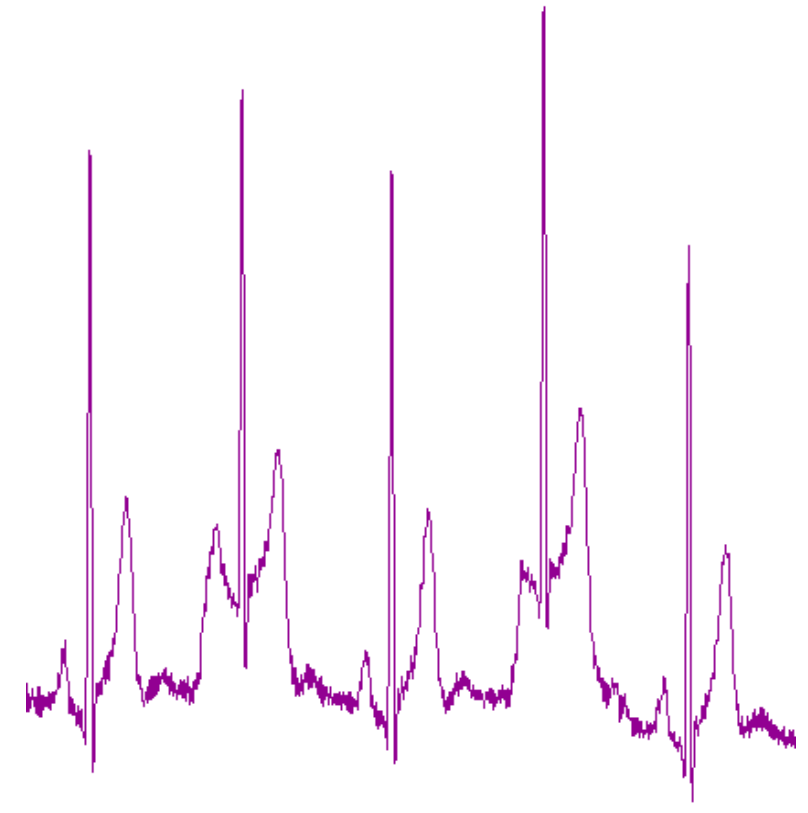

Fig 2:- De-Noised Signal \#1 (MATLAB)

Frequency - 100 cycles / 1 sec

Standard Deviation - 20 metres

Signal Accuracy - 89\%

Noise Removed $-\mathbf{9 2 \%}$

\subsubsection{Signal \#2}

Signal \#2 contains information which pertains to the data sent by locators. Locators are the devices which can locate entities that are either lost or not cannot be tracked with Trackers.

\section{Original Signal}

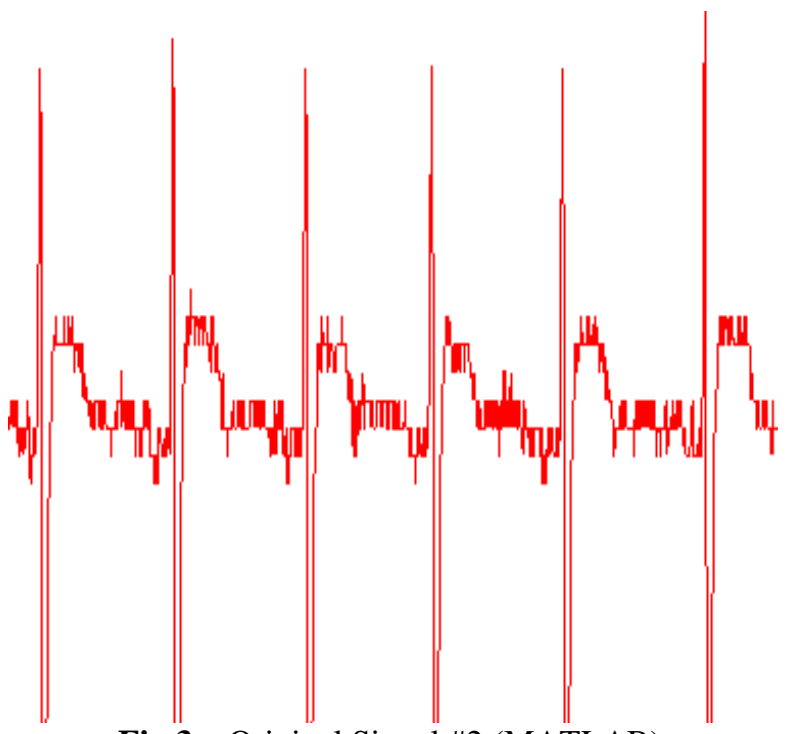

Fig 3 - Original Signal \#2 (MATLAB)

Frequency -130 cycles / 1 sec Standard Deviation - 15 metres

\section{De-Noised Signal}

A Decomposition Level - 2 has been used with Haar Wavelet Discrete Transformation and Inverse Discrete Transformation.

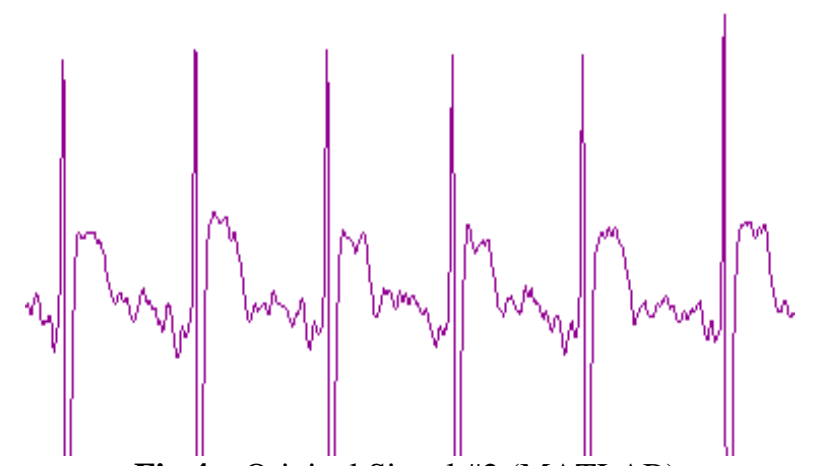

Fig 4 - Original Signal \#2 (MATLAB)

Frequency -130 cycles / 1 sec

Standard Deviation - 15 metres

Signal Accuracy - $93 \%$

Noise Removed - $91 \%$

\subsubsection{Signal \#3}

Signal \#3 contains information which pertains to the data sent by communicators. These signals carry very private information and so, the signals are secured with algorithms. 


\section{Original Signal}

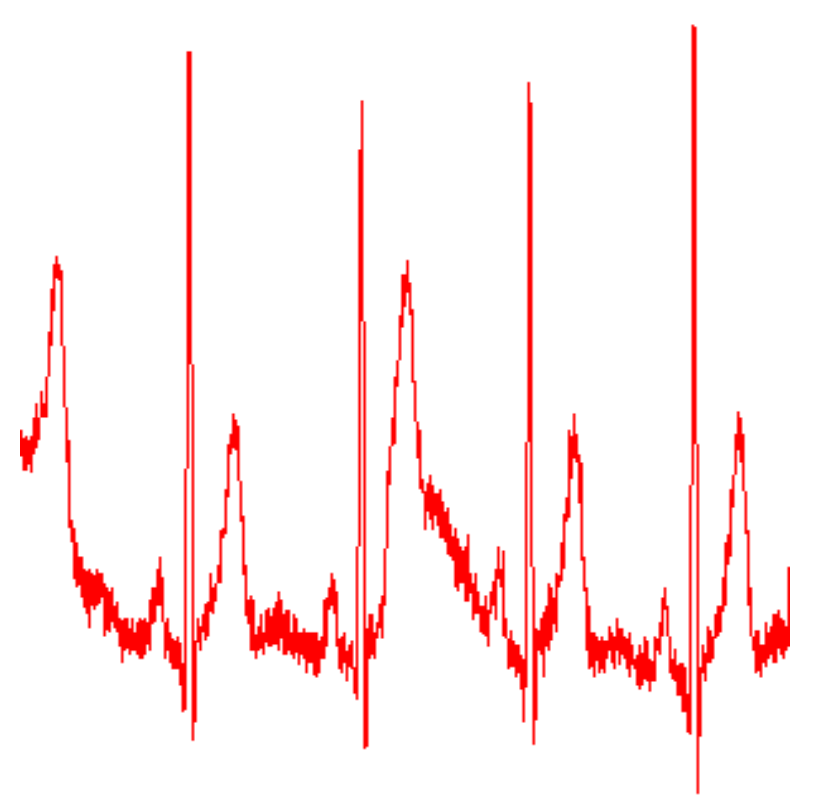

Fig 5 - Original Signal \#3 (MATLAB)

Frequency - 100 cycles / 1 sec

Standard Deviation - 20 metres

\section{De-Noised Signal}

A Decomposition Level - 2 has been used with Haar Wavelet Discrete Transformation and Inverse Discrete Transformation.

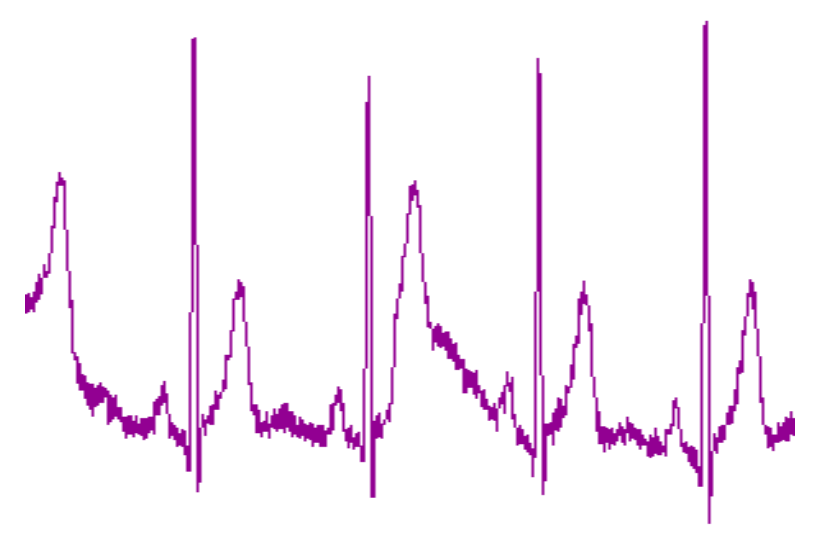

Fig 6 - Original Signal \#3 (MATLAB)

Frequency -100 cycles / 1 sec

Standard Deviation - 20 metres

Signal Accuracy - 97\%

Noise Removed $-\mathbf{8 9} \%$

\subsubsection{Signal \#4}

Signal \#4 contains information which pertains to the medical data of one staff member recorded internally.

\section{Original Signal}

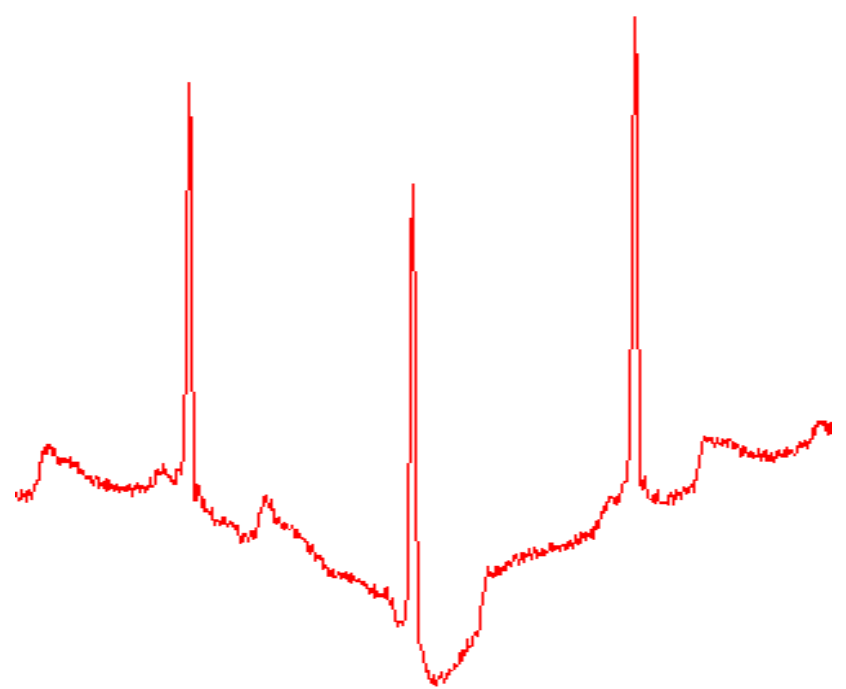

Fig 7 - Original Signal \#4 (MATLAB)

Frequency - 75 cycles / 1 sec

Standard Deviation - 30 metres

\section{De-Noised Signal}

A Decomposition Level - 2 has been used with Haar Wavelet Discrete Transformation and Inverse Discrete Transformation.

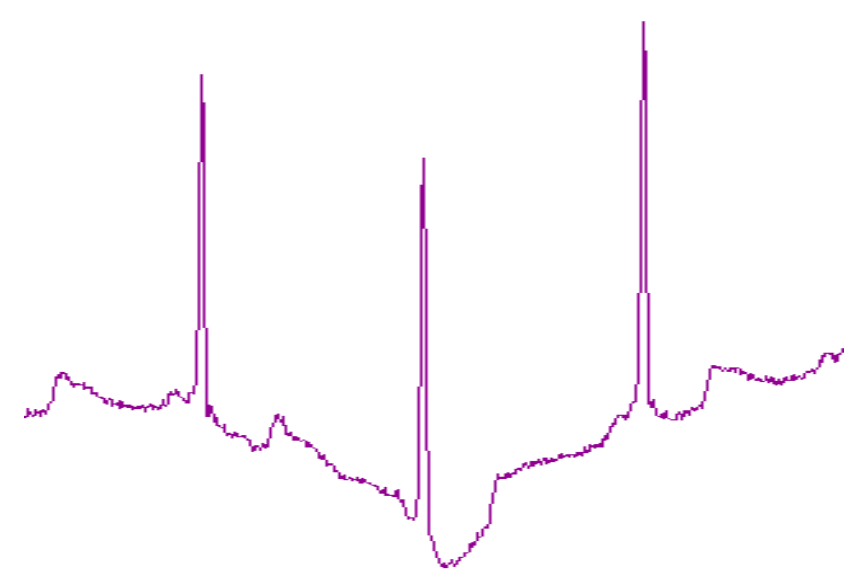

Fig 8 - Original Signal \#4 (MATLAB)

Frequency - 75 cycles / 1 sec

Standard Deviation - 30 metres

Signal Accuracy - 99\%

Noise Removed $-\mathbf{8 9 \%}$

\subsection{Python Tool Observations}

All the Observations using Python Tool have been conducted through the use of a custom, specially developed and designed UI module. The Signal Graphs shown below are only screenshots of Python Tool signal processing results. 


\subsubsection{Signal \#1}

Signal \#1 contains information which pertains to the data sent by trackers. Trackers are basic identifying devices which can be used to track any object.

\section{Original Signal}

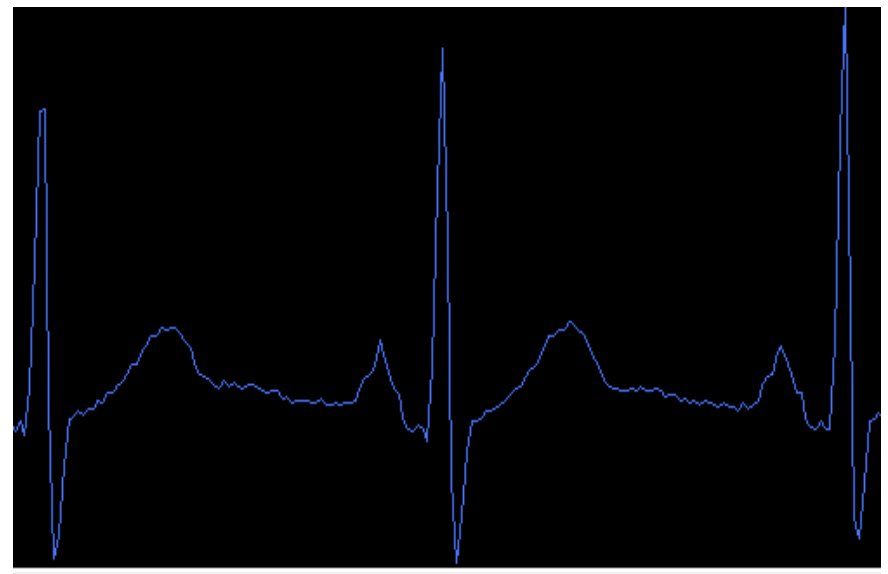

Fig 9 - Original Signal \#1 (PYTHON)

Frequency - 100 cycles / 1 sec

Standard Deviation - 20 metres

\section{De-Noised Signal}

A Decomposition Level - 2 has been used with Haar Wavelet Discrete Transformation and Inverse Discrete Transformation.

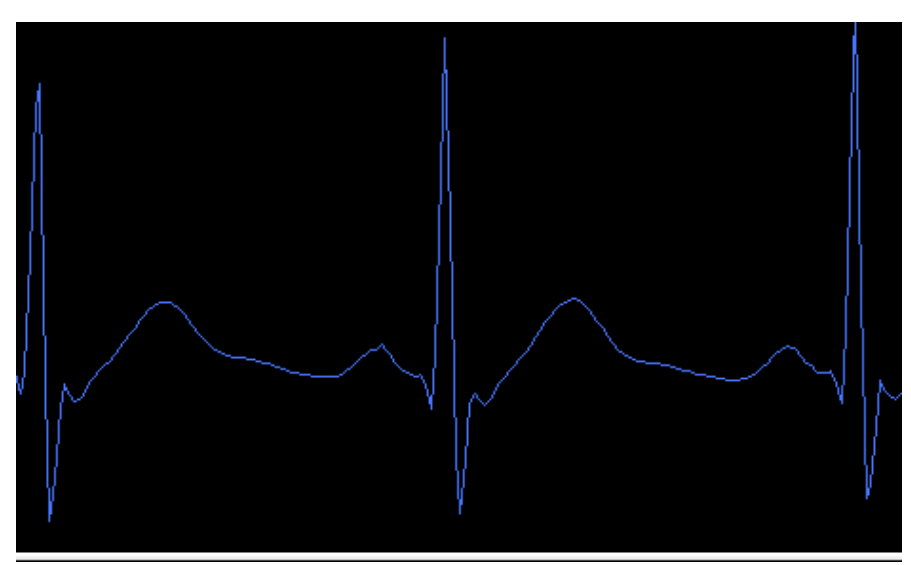

Fig 10 - De-Noised Signal \#1 (PYTHON)

Frequency - 100 cycles / 1 sec

Standard Deviation - 20 metres

Signal Accuracy - $\mathbf{9 0 \%}$

Noise Removed $-\mathbf{8 0} \%$

\subsubsection{Signal \#2}

Signal \#2 contains information which pertains to the data sent by locators. Locators are the devices which can locate entities that are either lost or not cannot be tracked with Trackers.

\section{Original Signal}

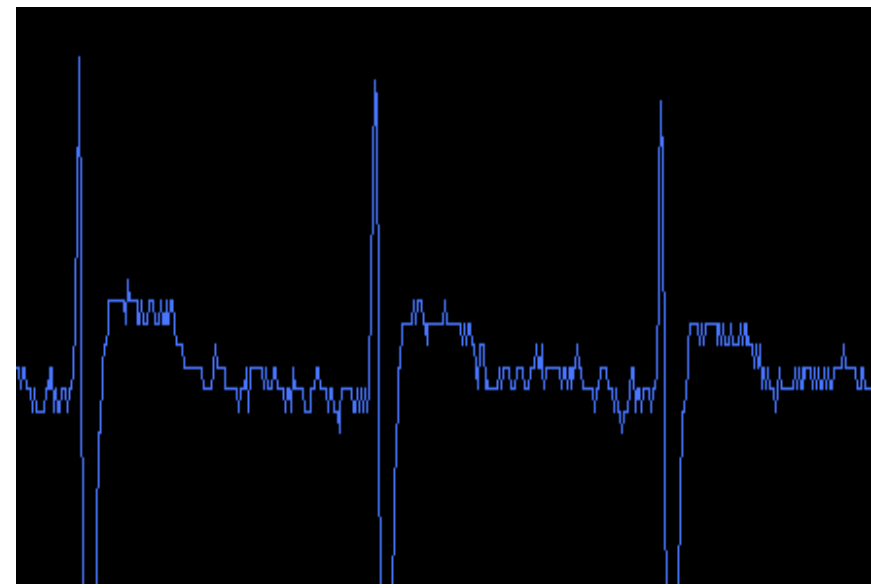

Fig 11 - Original Signal \#2 (PYTHON)

Frequency - 130 cycles / 1 sec

Standard Deviation - 15 metres

\section{De-Noised Signal}

A Decomposition Level - 2 has been used with Haar Wavelet Discrete Transformation and Inverse Discrete Transformation.

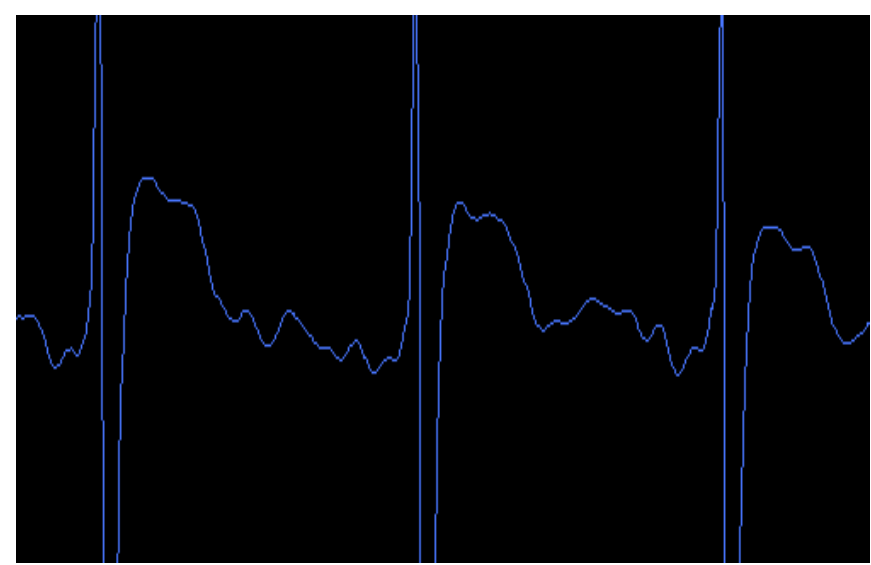

Fig 12 - De-Noised Signal \#2 (PYTHON)

Frequency - 130 cycles / 1 sec

Standard Deviation - 15 metres

Signal Accuracy $-\mathbf{1 0 0} \%$

Noise Removed - $95 \%$

\subsubsection{Signal \#3}

Signal \#3 contains information which pertains to the data sent by communicators. These signals carry very private information and so, the signals are secured with algorithms. 


\section{Original Signal}

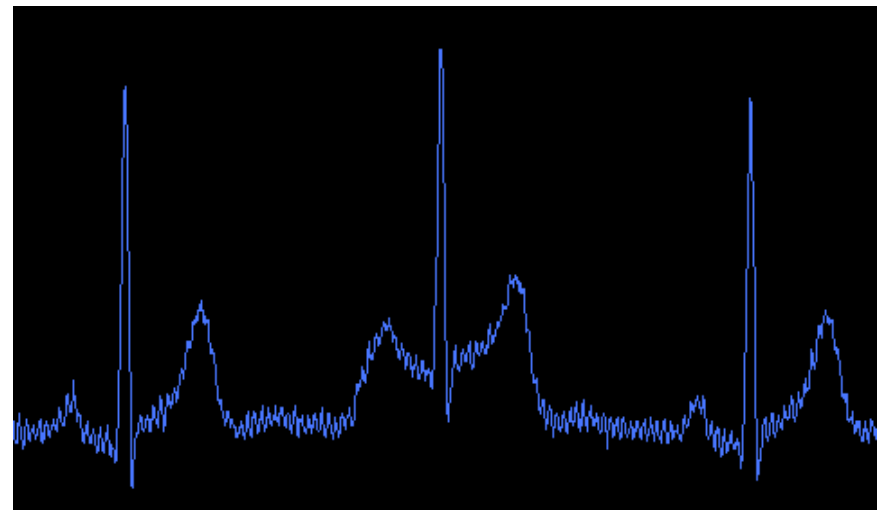

Fig 13 - Original Signal \#3 (PYTHON)

\section{Frequency - 100 cycles / 1 sec}

Standard Deviation - 20 metres

\section{De-Noised Signal}

A Decomposition Level - 2 has been used with Haar Wavelet Discrete Transformation and Inverse Discrete Transformation.

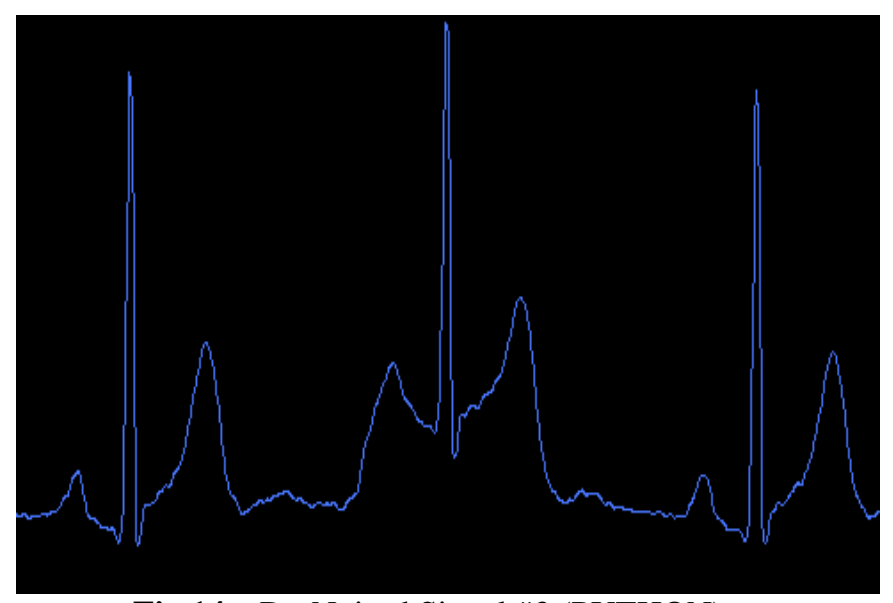

Fig 14 - De-Noised Signal \#3 (PYTHON)

Frequency - 100 cycles / 1 sec

Standard Deviation - 20 metres

Signal Accuracy - $\mathbf{9 0 \%}$

Noise Removed $-\mathbf{8 0 \%}$

\subsubsection{Signal \#4}

Signal \#4 contains information which pertains to the medical data of one staff member recorded internally.

\section{Original Signal}

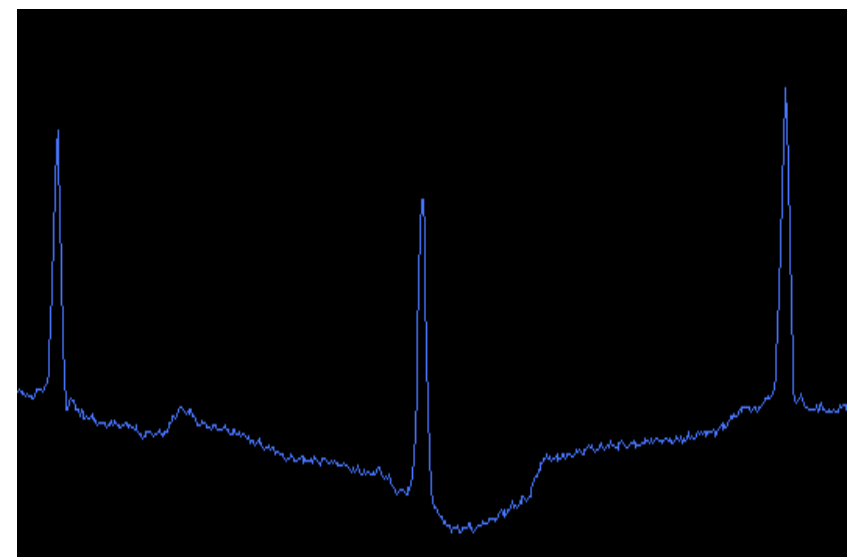

Fig 15 - Original Signal \#4 (PYTHON)

Frequency -75 cycles / 1 sec

Standard Deviation - 30 metres

\section{De-Noised Signal}

A Decomposition Level - 2 has been used with Haar Wavelet Discrete Transformation and Inverse Discrete Transformation.

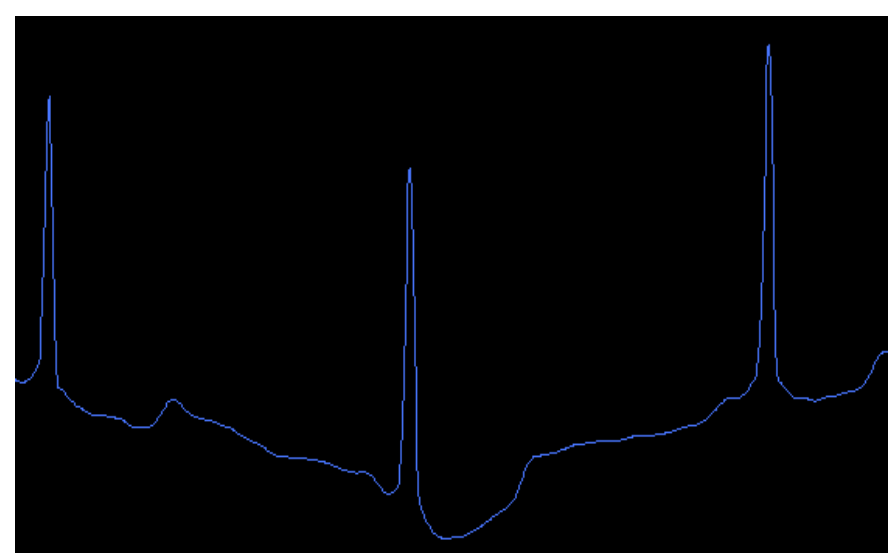

Fig 16 - De-Noised Signal \#4 (PYTHON)

Frequency - 75 cycles / 1 sec

Standard Deviation - 30 metres

Signal Accuracy - 95\%

Noise Removed $-\mathbf{9 0 \%}$

\subsection{Analysis}

The results obtained from above observational cases are recorded in the tables below for comparison purposes and to determine the most accurate and efficient tool in data signal de-noising. The comparative value tables are as follows:

\begin{tabular}{|l|l|l|l|l|}
\hline Attribute & $\begin{array}{l}\text { Signal } \\
\mathbf{\# 1}\end{array}$ & $\begin{array}{l}\text { Signal } \\
\mathbf{\# 2}\end{array}$ & $\begin{array}{l}\text { Signal } \\
\mathbf{\# 3}\end{array}$ & $\begin{array}{l}\text { Signal } \\
\mathbf{\# 4}\end{array}$ \\
\hline $\begin{array}{l}\text { Frequenc } \\
\mathbf{y}\end{array}$ & 100 & 130 & 100 & 75 \\
\hline Stnd. Dev. & 20 & 15 & 20 & 30 \\
\hline
\end{tabular}




\section{Matlab Specifics}

\begin{tabular}{|l|l|l|l|l|}
\hline Signal Acc. & $89 \%$ & $93 \%$ & $97 \%$ & $99 \%$ \\
\hline Noise Rem. & $92 \%$ & $91 \%$ & $89 \%$ & $89 \%$ \\
\hline
\end{tabular}

Observation - Very Consistent with colour indentation, average efficiency but high cost and high memory requirement.

\section{Python Tool Specifics}

\begin{tabular}{|l|l|l|l|l|}
\hline Signal Acc. & $90 \%$ & $100 \%$ & $90 \%$ & $95 \%$ \\
\hline Noise Rem. & $80 \%$ & $95 \%$ & $80 \%$ & $90 \%$ \\
\hline
\end{tabular}

Observation - Very Efficient, cost-effective and memory friendly but no colour indentation and bad consistency.

Frequency - Number of cycles present in ONE second time.

Stnd. Dev. - Standard Deviation (Average Deviation between crusts).

Signal Acc. - Percentage of signal accuracy when compared to original signal.

Noise Rem. - Percentage of Noise Removed from original signal.

\section{RESULTS and DISCUSSIONS}

The most important purpose of these analytics is to guide schemata of de-noising techniques available in two different tools, which are: MATLAB and Python Tool. We have given four same signals as input to the two tools and we were able to ascertain their efficiency, obtain their consistency, observe their performance, note their memory taxation and finally, we were also able to see their relative cost expenses. After the aforementioned comparative critical analysis of all the involved components, we have arrived at a conclusive result.

In all our experiments and testing with MATLAB using 4 signals, we have noticed that MATLAB is very consistent and easy to understand due to colour indentation, but is also costly both monetarily and memory wise. Efficiency in denoising and retaining relevant data is found to be average.

As of our experiments and testing with Python Tool using 4 signals, we have observed that our Python Tool is very efficient, almost takes $25 \%$ of memory taken by MATLAB and projected monetary cost at maintaining is also very low. Consistency is a bit lacking feature in our Python tool and it may be a bit harder to understand in first look due to lack of specialised colour indentation.

Overall, if the client requirement is data signal de-noising efficiency and maintenance cum memory cost effectiveness, then our PYTHON TOOL is optimal. If the client requirement is consistency and ease of understanding, then MATLAB TOOL is optimal.

\section{REFERENCES}

(All the references were accessed during Feb-March 2015). [1]. Introduction to Haar Wavelets - Portland State University.

[2]. Besslich PhW, Trachtenberg LA. The sign transform: an invertible non-linear transform with quantized coefficients. In: Moraga $\mathrm{C}$, editor. Theory and application of spectral techniques. University Dortmund Press; October 1988.

[3]. Beauchamp KG. Applications of Walsh and related functions with an introduction to sequency theory. New York: Academic Press and Publications p. 4-13; 1984.

[4]. Boiko LL. Generalized Fourier-Haar transform on finite Abelian groups. In: Yaroslavsky LP, editor. Digital signal processing and applications. Moscow: Nauka; 1981. p. $12-22$

[5]. Buron AM, Michell JA, Sloana M. Single chip fast Haar transform at megahertz rates. In: Moraga C, editor. Theory and application of spectral techniques. University Dortmund Press and publications for open source; October 1988, p. 8-17.

[6]. Burrus CS, Gophinat RA, Guo H. Introduction to wavelets and wavelet transforms. Englewood Cliffs: Prentice Hall; 1988.

[7]. Castleman KR. Digital image processing. Englewood Cliffs Press and publications p. 13-22: Prentice-Hall; 1996. [8].https://en.wikipedia.org/wiki/Haar_Transformation_wa velet

[9].https://en.wikipedia.org/wiki/Discrete_wavelet_transfor $\mathrm{m}$

\section{BIOGRAPHIES}

1. Ananth Abhishek Jillepalli, IV ${ }^{\text {th }}$ Year, Department of CSE, Vidya Jyothi Institute of Technology, Hyderabad.

E-Mail: ananth9328@gmail.com

2. Raghavendra Deeduvanu, $\mathrm{IV}^{\text {th }}$ Year, Department of CSE, Vidya Jyothi Institute of Technology, Hyderabad.

E-Mail: raghavendra065@gmail.com

3. Sreenath DeviReddy, $\mathrm{IV}^{\text {th }}$ Year, Department of CSE, Vidya Jyothi Institute of Technology, Hyderabad.

E-Mail: sreenathreddy9194@gmail.com

4. Deepak Vangala IV $^{\text {th }}$ Year, Department of CSE, Vidya Jyothi Institute of Technology, Hyderabad.

E-Mail: deep.vangala21@gmail.com

5. Ravi Mathey, Head of Department, Department of Computer Science and Engineering, Vidya Jyothi Institute of Technology, Hyderabad.

E-Mail: ravimathey@gmail.com 